\title{
Irreversible Effects of Ivermectin on Adult Parasites in Onchocerciasis Patients in the Onchocerciasis Control Programme in West Africa
}

\author{
Anton P. Plaisier, E. Soumbey Alley, Boakye A. Boatin, \\ Gerrit J. Van Oortmarssen, Hans Remme, \\ Sake J. De Vlas, Luc Bonneux, and \\ J. Dik F. Habbema
}

\begin{abstract}
Centre for Decision Sciences in Tropical Disease Control, Department of Public Health, Faculty of Medicine, Erasmus University Rotterdam, Netherlands: Onchocerciasis Control Programme in West Africa, Ouagadougou, Burkina Faso; United Nations Development Program/ World Bank/World Health Organization Special Program for Research and Training in Tropical Diseases, World Health Organization,
\end{abstract}

Geneva, Switzerland

\begin{abstract}
Ivermectin is an effective drug for the treatment of human onchocerciasis, a disease caused by the parasitic filarial nematode Onchocerca volvulus. When humans are treated, the microfilariae normally found in the skin are rapidly and very nearly completely eliminated. Nonetheless, after a delay, microfilariae gradually reappear in the skin. This study is concerned with the causes of this delay. Hypotheses are tested by comparing the results of model calculations with skin microfilaria counts collected from 114 patients during a trial of five annual treatments in the focus area of Asubende, Ghana. The results obtained strongly suggest that annual treatment with ivermectin causes an irreversible decline in microfilariae production of $\sim 30 \% /$ treatment. This result has important implications for public health strategies designed to eliminate onchocerciasis as a significant health hazard.
\end{abstract}

The registration of the anthelminthic drug ivermectin (Mectizan; Merck, Rahway, NJ) in 1987 was a landmark in the control of human onchocerciasis or river blindness, a parasitic disease caused by the filarial nematode Onchocerca volvulus. Oral administration in a standard dose of $150-200 \mu \mathrm{g} / \mathrm{kg}$ of body weight is followed by rapid elimination of microfilariae (Mf) from the skin and gradual reduction of ocular Mf levels [1]. Side effects are generally mild. This makes ivermectin a better therapeutic option than diethylcarbamazine, which is often accompanied by severe Mazotti reactions and ocular damage. Ivermectin also produces a longer suppression of $\mathrm{Mf}$ repopulation of the skin $[2,3]$. To explain this difference, which was obvious in all studies done so far, the effects of ivermectin on adult parasites were studied.

Adult female parasites in treated persons show an interruption of the normal embryogenesis, but after a single treatment, this appears to be reversible for most of the worms $[4,5]$. Excess worm mortality was not observed after a single treatment $[1,2,6]$, although significant numbers of dead and mori-

Received 5 July 1994; revised 14 February 1995.

All subjects gave informed consent. The treatment and monitoring protocol was approved by Onchocerciasis Control Programme in West Africa (OCP), Ouagadougou, Burkina Faso; Onchocerciasis Chemotherapeutic Research Centre, Tamale, Ghana; and Ministry of Health of Ghana.

Financial support: Technical Service Agreement, World Health Organization, on behalf of OCP

Reprints or correspondence: Dr. A. P. Plaisier, Centre for Decision Sciences in Tropical Disease Control, Dept. of Public Health, Faculty of Medicine, Erasmus University Rotterdam, P.O. Box 1738, 3000 DR Rotterdam, Netherlands.

The Journal of Infectious Diseases $1995 ; 172: 204-10$

(C) 1995 by The University of Chicago. All rights reserved.

$0022-1899 / 95 / 7201-0028 \$ 01.00$ bund worms were reported after multiple treatments at intervals between 2 weeks and 6 months [6-11]. Furthermore, after multiple treatments, the reproductive activity of the surviving worms was markedly reduced. The suppression of Mf production persisted 1.5 years after five 6-monthly doses [12], which suggests the existence of irreversible effects of the drug.

An important question in view of the limited resources available to health services in developing countries is whether these findings for shorter treatment intervals are applicable to regimens with intervals of 1 year, the current practice in nearly all onchocerciasis control programs [13]. In this study, we addressed this question by analyzing data from a large community-based study of annual treatment in a region with hyperendemic onchocerciasis (Asubende, Ghana), organized by the Onchocerciasis Control Programme in West Africa (OCP) [14-17].

The available data consist of Mf counts in skin snips from persons who were repeatedly surveyed over $\sim 5$ years during treatment. We investigated whether the observed changes in Mf counts could be explained if ivermectin had only transient effects on adult worms or whether long-term irreversible effects on the fecundity of adult worms were required to explain the data. In addition to the direct effect in individual patients, community treatment with ivermectin also reduces the level of transmission in the area (indirect effect) $[14,17]$. To take both effects into account, we did the investigation with the aid of an epidemiologic model of onchocerciasis transmission.

\section{Material and Methods}

Study population. The Asubende region is along the lower reaches of the Pru River, just west of Lake Volta. OCP initiated a 
program to control the vector of $O$. volvulus, the blackfly Simulium damnosum, in this river basin in January 1986. Flies have been collected since 1979 to assess the vector biting rate and the vector infectivity. A clinical survey was done in September 1987 among 796 persons living in a cluster of three villages in the middle of the area. Both entomologic and clinical findings revealed that the savanna form of $O$. volvulus is hyperendemic in this area. Skin Mf densities were among the highest encountered in the OCP area [14]. The community trial of annual ivermectin treatment was started in October 1987.

In the present analysis we use data consisting of counts of Mf in skin snips that were collected during the period covered by the first five treatments (1987-1991). The organization of the trial, the trends in skin Mf densities, and the joint effect of vector control and ivermectin treatment on the transmission potential of the flies have been described elsewhere $[14,17]$. Treatment dose varied between 130 and $200 \mu \mathrm{g} / \mathrm{kg}$.

From the 796 persons examined at the baseline survey in 1987 , we selected 2 cohorts that comprised a total of 114 adults. Cohort 1 is 78 persons who were treated in all five rounds and were examined in all eight follow-up surveys. These surveys were done at 4 and 12 months after the first two treatments, 5 and 12 months after the third, 11 months after the fourth, and 6 months after the fifth treatment. Cohort 2 is 36 persons who were treated in the first but not in the second round and who were examined 24 months after the first treatment. We restricted the study to adults to exclude the confounding effect of ageing; only for older ages do we expect a constant infection level [18].

Model and hypotheses. We tested two hypotheses: The first $\left(\mathrm{H}_{1}\right)$ assumes that treatment has only a transient effect on the Mf production of female worms; the second $\left(\mathrm{H}_{2}\right)$ assumes that ivermectin's effect is partially irreversible, manifested by a permanently reduced fecundity of the worms in a treated patient. Hypotheses were tested with the stochastic microsimulation model ONCHOSIM. This model simulates the life histories of hypothetical individual persons (birth, acquisition of parasites, death) and individual parasites (maturation, mating, production of $\mathrm{Mf}$, death) and can be used to describe and test effects of ivermectin on a patient level. ONCHOSIM further allows for a detailed simulation of control strategies. The model simulates ivermectin treatments and surveys at the same times as in the field. It also mimics the vector control activities used in the Asubende area, which strongly affected the transmission potential of the flies both before and during the trial [17]. The microsimulation method makes it possible to select persons from the simulated population who satisfy the same criteria used for selecting the cohorts and whose Mf counts before the first treatment are the same as those of the cohorts.

A full description of ONCHOSIM and the validation of the model is given elsewhere [19-22]. Here we will present only those rclationships and parameters that are directly relevant to the effects of ivermectin.

Modeling the effects of ivermectin. An assumption used throughout the analysis is that a treatment with ivermectin instantly eliminates all $\mathrm{Mf}$ in a person, except in $3 \%$ of treatments, which totally fail as a result of malabsorption (e.g., diarrhea, vomiting) [23]. Under the first hypothesis $\left(\mathrm{H}_{1}\right)$, each treatment further causes a transient interruption of the normal release of $\mathrm{Mf}$ by adult female parasites. During a recovery period, the rate of Mf production increases from zero to the pretreatment level. We explicitly test whether this increase is linear or not.

The same assumption regarding the initial effect of treatment is made under the alternative hypothesis $\left(\mathrm{H}_{2}\right)$. However, in this case, we assume that worms do not recover to their full capacity to release $\mathrm{Mf}$, but that this capacity is irreversibly reduced with a fraction called "productivity reduction." This productivity reduction affects all worms in a patient.

Since the available data do not reveal much about the exact mechanism of a (possible) irreversible effect, we also tested an alternative form of the second hypothesis $\left(\mathrm{H}_{2}^{\prime}\right)$, in which a certain fraction of worms loses their fecundity totally, while the others recover completely. This fraction is called "fecundity loss."

Between-treatment (and within-patient) variation in recovery period, productivity reduction, and fecundity loss is governed by effect variability, defined as the variation coefficient (i.c., the quotient of SD and mean) of treatment effects. The time between treatment and the stabilization of the Mf density in skin is determined not only by the effect of ivermectin on adult worms but also by the life span of Mf. This Mf life span is estimated when the hypotheses are tested. A mathematical description of the submodel for the effect of ivermectin treatment is given in the Appendix. A graphic representation of the hypotheses and roles of the model parameters are provided in figure 1 .

Hypothesis testing. To test hypotheses and quantify model parameters, both observed and calculated survey results were represented as frequency distributions of $\mathrm{Mf}$ counts, using the classes shown in figure 2 . The agreement between observations and predictions was determined by calculating a total $\chi^{2}$ for all follow-up surveys of cohorts 1 and 2. Some adjacent skin snip count classes were combined so that the number of individuals was at least 5/ class. Estimating parameters for a specific hypothesis was achieved by minimizing $\chi^{2}$ using a downhill simplex method [24]. The goodness-of-fit implied by the minimum $\chi^{2}$ was quantified by the $P$ value of the $\chi^{2}$ distribution with $16 d f$ (only transient effects) or $15 d f$ (also irreversible effects). Generally, $P<.05$ indicates a poor fit and suggests rejection of the underlying hypothesis.

After model parameters were estimated, a $95 \%$ confidence interval (CI) of a particular estimate was determined by searching around the original estimate for values resulting in $\chi^{2}=3.84$ units $\left(0.95\right.$ point of $\chi^{2}$ distribution with $\left.1 d f\right)$ higher than the minimum. For each of the values tried, the other ivermectin-related parameters were reestimated by minimizing $\chi^{2}$.

\section{Results}

For the baseline and follow-up surveys, the number of Mf per skin snip observed in the 2 cohorts are shown in the frequency distributions in figure 2. Considering the data from cohort 1 , both short-term and longer-lasting effects of treatment were apparent. At 4-6 months after each treatment, the distribution was consistently skewed toward low Mf counts. Although after 11-12 months the distributions again tended to the pretreatment shape, it is clear that, as the number of treatments increased, the peak of the distributions shifted to lower counts: $\sim 32-64 \mathrm{Mf} / \mathrm{snip} 1$ year after the first treatment to $4-16 \mathrm{Mf} /$ snip 1 year after the fourth round. A long-term effect was also 

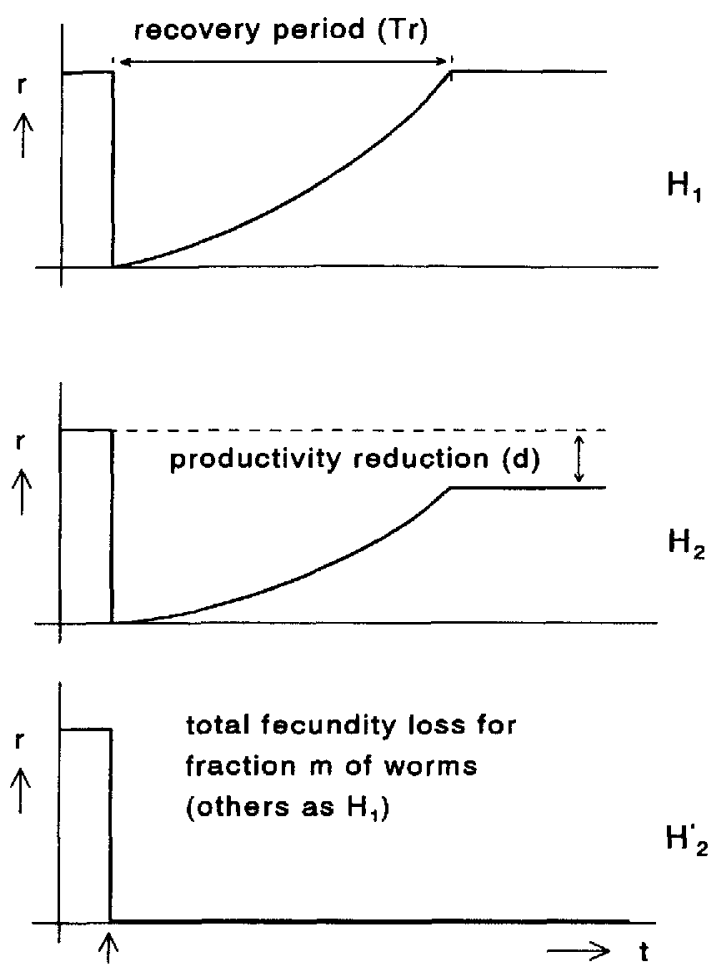

Figure 1. Schematic representation of hypotheses tested. Each graph shows rate of microfilaria production $(r)$ of parasite as function of time $(t)$ after single treatment. Moment of treatment is indicated with vertical arrow below $x$ axis. In hypothesis $\mathrm{H}_{1}$, no irreversible effect is assumed; treatment only results in recovery period of length $T r$. According to hypothesis $\mathrm{H}_{2}$, productivity after recovery period is permanently reduced (factor $d$ ); with $\mathrm{H}_{2}{ }^{\prime}$, fraction $m$ of worms totally loses fecundity. See Appendix for further explanation of symbols.

observed in the data from cohort 2 (figure 2B); before treatment, $\sim 30 \%$ of the population had $>128 \mathrm{Mf} / \mathrm{skin}$ snip. Two years after a single treatment this was only slightly $>15 \%$.

We tested two hypotheses for explaining the data (figure 1). The results are shown in table 1. For each hypothesis, estimates of relevant parameters and goodness-of-fit ( $P$ values) are given. $\mathrm{H}_{1}$, which assumed no irreversible effects of ivermectin, fits poorly $(P=.0013)$ and must be rejected. By contrast, a good fit was obtained when an irreversible productivity reduction for all worms was considered $\left(\mathrm{H}_{2}: P=.68\right)$. This productivity reduction was $\sim 35 \% /$ treatment $(95 \% \mathrm{CI}, 26 \%-40 \%)$ ). Assuming another mechanism for the irreversible effect of treatment $\left(\mathrm{H}_{2}{ }^{\prime}\right)$ did not lead to significantly different conclusions. Total fecundity loss was $\sim 28 \%$ /treatment, while the other parameter estimates were about the same for $\mathrm{H}_{2}$ and $\mathrm{H}_{2}{ }^{\prime}$. A model that combines both types of irreversible effect (not shown) did not produce a significantly better fit to the data. In such a model, the combined loss of Mf production in a patient was $\sim 32 \%$ / treatment $(95 \% \mathrm{CI}, 22 \%-40 \%)$.

The irreversibly reduced level of Mf production was preceded by a recovery period of $\sim 10$ months during which the production rate accelerated (see the nonlinear trends in figure $1 ; s=1.5$, Appendix). Both the recovery period and the Mf life span are inversely related to the speed of the Mf repopulation of the skin after treatment. Therefore, apparently to compensate for the lack of irreversible effect, under the first hypothesis, both parameters were estimated at significantly higher values.

The effect of treatment varied considerably between treatments. The value of 0.52 estimated for effect variability $\left(\mathrm{H}_{2}\right)$ means that one-fourth of treatments resulted in a recovery period of $<6$ months and a productivity reduction of $<20 \%$. By contrast, for another one-fourth these numbers were $>14$ months and $>47 \%$, respectively. Half of treatments had an intermediate efficacy.

Model results, assuming irreversible effects, were compared with the observations (figure 2, open bars). There were no systematic differences between prediction and observations for cohort 1 (figure 2A). The lowest and highest Mf count categories of the follow-up survey of cohort 2 were underestimated (figure 2B), suggesting more treatment variability in this cohort. For cohort 1, model predictions under both hypotheses are shown in figure 3. Here, predictions and observations arc represented as the geometric mean Mf count. Only the pretreatment and follow-up surveys at 11-12 months are shown (i.e., just before another treatment). Under both hypotheses, the geometric mean Mf counts decline sharply after the first treatment and more gradually thereafter. However, the slope of the line connecting the follow-up surveys is much steeper if irreversible changes are considered $\left(\mathrm{H}_{2}\right)$. Under $\mathrm{H}_{1}$, the Mf counts after repeated treatment decline only because the transmission dynamics of the infection are disturbed by the combination of treatment and vector control. This decline is too slow to fit the observations.

\section{Discussion}

Our analysis of the results of five consecutive annual treatments provides strong evidence that apart from killing Mf, ivermectin reduces the viability of adult female parasites. The trend in Mf counts was explained by the hypothesis that after each treatment, Mf production gradually increases over 10 months (transient effect) and then reaches a plateau that remains $32 \%$ lower than before treatment (irreversible effect; combining $\mathrm{H}_{2}$ and $\mathrm{H}_{2}{ }^{\prime}$ ).

The transient effect agrees with results of earlier studies, which showed that in most parasites, the normal release of Mf was interrupted after treatment, but 10-12 months later, Mf production had largely been restored $[4,5,25]$. Although none of these studies revealed excess mortality of worms from a single treatment, after 1 year a considerable fraction $(40 \%[5])$ was not (yet) releasing Mf, which explains the considerable delay in repopulation of the skin by Mf [1, 3, 26-28]. In some studies, even 2 years after treatment, Mf density was markedly reduced $[29,30]$, although most persons had become Mf-positive again. Indeed, in cohort 2 (treated only once), the mean 

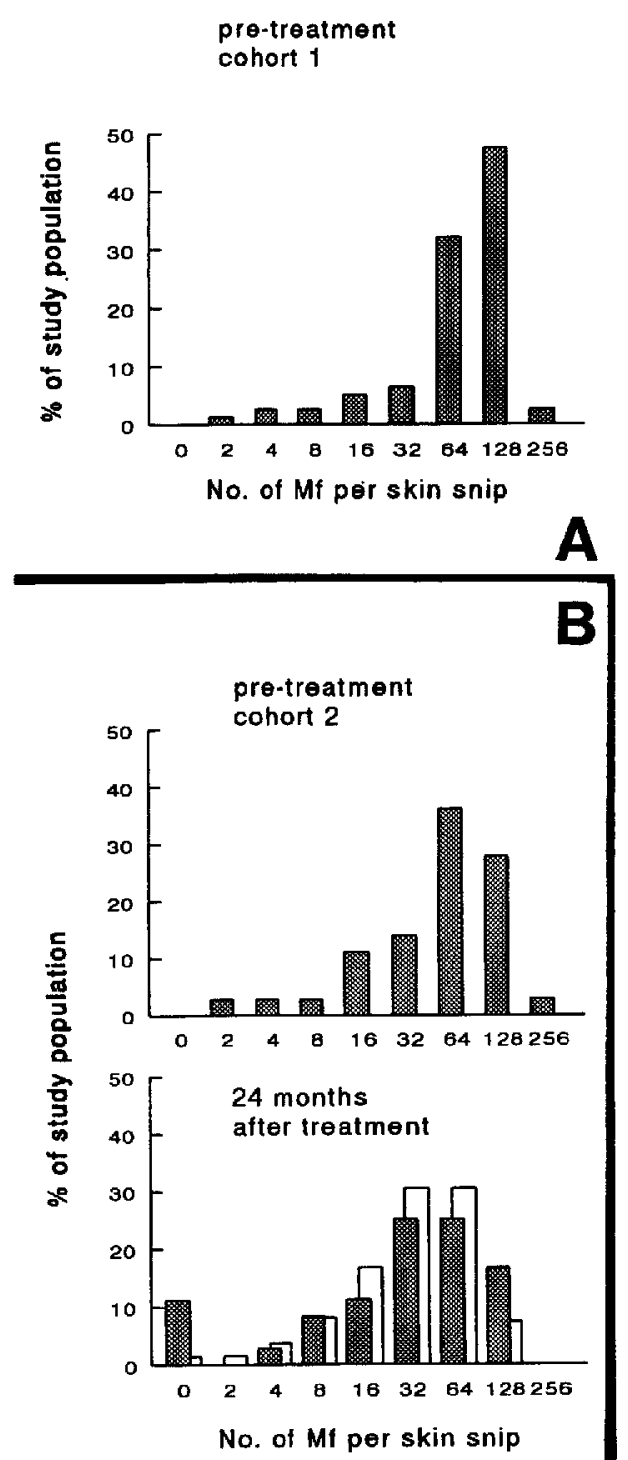

4-6 months after treatment
11-12 months after treatment treatment round
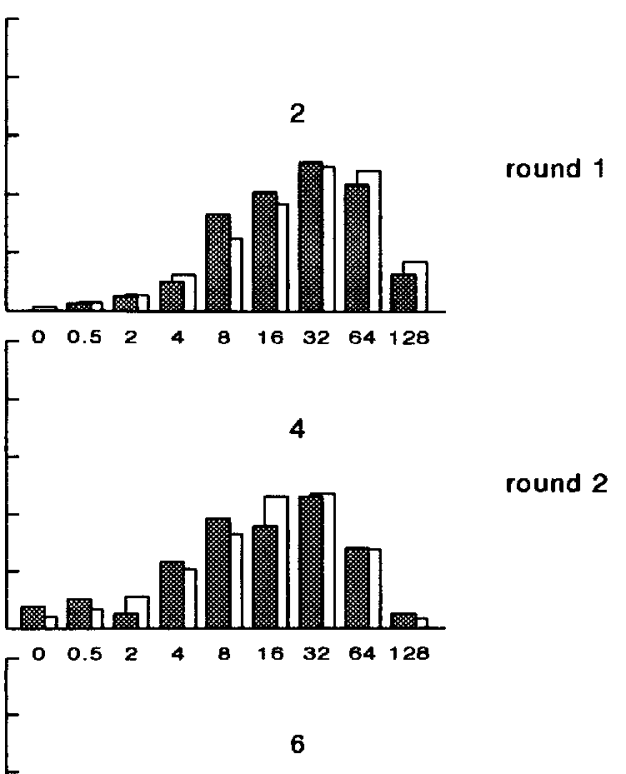

round 3
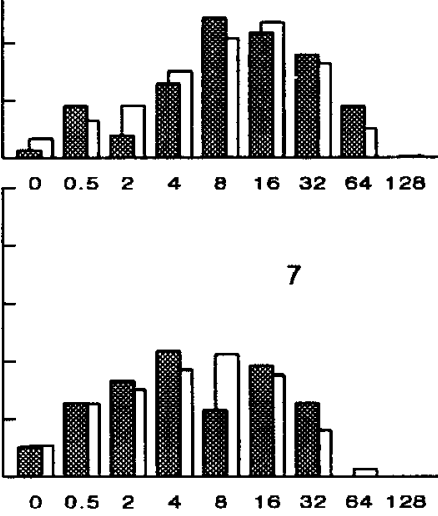

round $4 / 5$

Figure 2. Frequency distributions of microfilarial (Mf) counts for pre- and posttreatment surveys. A, Cohort 1,78 patients treated 5 times and examined 8 times. B, Cohort 2, 36 patients treated once and examined 24 months later. Shaded bars $=$ observations; open bars $=$ predictions based on hypothesis $\mathrm{H}_{2}$ (using parameter estimates of table 1 ); $x$ axes = lower boundaries of skin snip count classes; nos. in graphs = survey order.

Mf density after 2 years was still less than half the pretreatment level, suggesting a lasting effect.

On the other hand, as figure 3 makes clear, one must be careful in drawing conclusions from decreasing Mf trends. Also, without irreversible drug effects, the model predicts a decrease (albeit insufficient) in Mf count at the follow-up surveys at 11-12 months. This decrease is exclusively due to the reduced transmission of the parasite as a consequence of ivermectin treatment and (partial) vector control [17]. This effect on the transmission was taken into account under the assumption that the parasite larvae in flies originate from inhabitants of Asubende and will again be transmitted to them. Such an assumption would not be valid in areas with migration of fies, but Asubende is an isolated focus with a local transmission [14].

So far, irreversible effects of ivermectin have been observed only after multiple treatments at intervals of $\leqslant 6$ months. In patients who receive $4-12$ doses at intervals of 2 weeks to 6 months, more dead or moribund female parasites are found than in controls who receive no or only 1 treatment $[5-7,9$, 10]. Excess worm mortality of $25 \%-33 \%$ is observed after $8-$ 11 doses at 3-month intervals [9]. Though these are significant percentages, they are about equal to the irreversible effect we found for each round of treatment. This suggests that killing of worms is not the most important component of the irreversible effect: $25 \%$ excess mortality after 8 treatments implies (only) 
Table 1. Parameter estimates and goodness-of-fit for the different hypotheses about the effect of ivermectin on the microfilaria (Mf) production of adult parasites..

\begin{tabular}{lccc}
\hline & \multicolumn{3}{c}{ Hypothesis* } \\
\cline { 2 - 4 } Parameter & $\mathrm{H}_{1}$ & $\mathrm{H}_{2}$ & $\mathrm{H}_{2}^{\prime}$ \\
\hline Productivity reduction & - & $35 \%(26 \%-40 \%)$ & - \\
Total fecundity loss & - & - & $28 \%(22 \%-35 \%)$ \\
Recovery period (months) & 19.0 & $10.4(7-16)$ & 10.7 \\
$\begin{array}{l}\text { Effect variability (coefficient of } \\
\quad \text { variation) }\end{array}$ & 0.87 & $0.54(0.4-0.7)$ & 0.52 \\
Mf lifespan (months) & 14 & $9(4-12)$ & 10 \\
Goodness-of-fit $\left(P\left[\chi^{2}\right]\right)$ & $.0013[39]$ & $.68[11.9]$ & $.42[15.5]$ \\
\hline
\end{tabular}

NOTE. - , this parameter is not considered and thus has value of 0 . Nos. in parentheses are $95 \%$ confidence intervals.

* See legend of figure 1 for description of hypotheses.

$\sim 3.5 \%$ excess mortality per treatment. Furthermore, 6 months after the last dose (the longest follow-up in most studies), most female worms had not yet resumed normal embryogenesis and viability was markedly reduced. Recent investigations suggest that recovery to full productivity after this short follow-up period is unlikely or will take $>2$ years [12].

The available data on Mf counts do not allow us to draw inferences on the biologic mechanisms responsible for the irreversible treatment effect. The data are equally well fit by two quite different mechanisms: an average productivity reduction of $35 \%$ for all worms in a patient or the total loss of fecundity by an average of $28 \%$ of the worms. The difference between

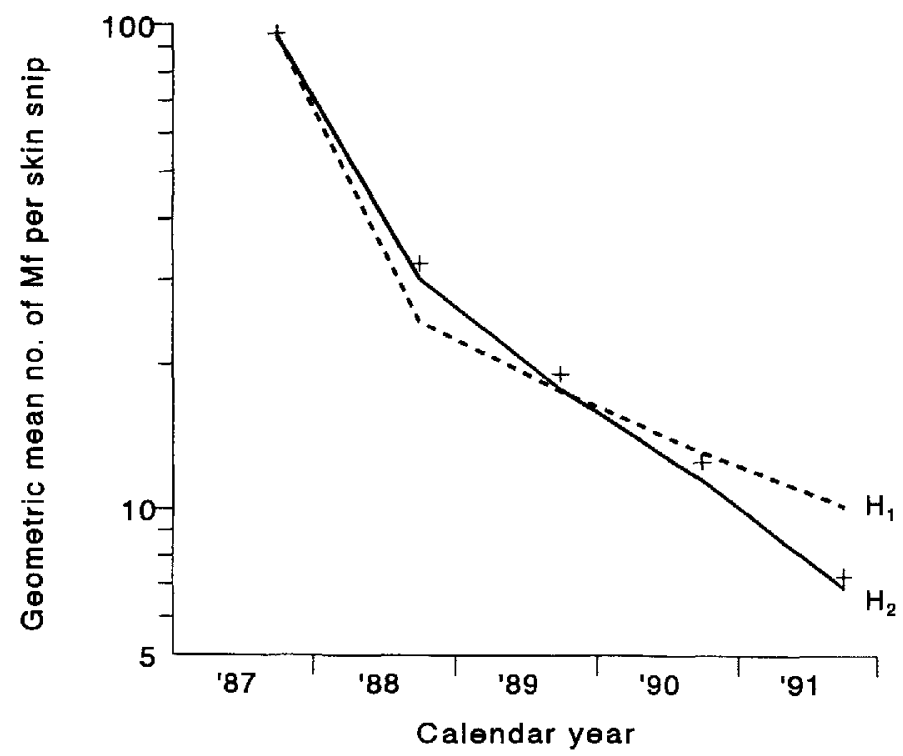

Figure 3. Observed $(+)$ and predicted geometric mean microfilaria (Mf) counts (log scale) for pretreatment and 11-12 follow-up surveys of cohort 1. Solid line = prediction based on hypothesis $\mathrm{H}_{2}$ (using parameter estimates of table 1 ); dashed line = prediction based on hypothesis $\mathrm{H}_{1}$. these estimates is caused by the different impact of the two mechanisms on the Mf counts in persons with low worm loads. If each treatment eliminates a certain fraction of productive worms, then in such persons frequently no productive worm is left (especially after repeated treatment) and, as a consequence, the skin snip is negative (no Mf). In the simulated population this leads to more skin snip-negative persons than with an equal percentage of productivity reduction. This explains the (slightly) lower estimate for fecundity loss.

Other mechanisms also could cause both transient and irreversible changes. For example, some studies demonstrate a significant reduction of the number of male worms [7, 9]. Although these lower counts may be the result of the ability of male worms to leave nodules [31], it may at least temporarily lead to reduced mating chances and a lower Mf output of female worms. Hence, we cannot exclude that part of the effect estimated for female worms should be attributed to males. It is important to stress that the results of our analysis are based on data collected during annual treatment using a dose of $130-$ $200 \mu \mathrm{g} / \mathrm{kg}$. Changes in treatment frequency or dose may lead to other effects per treatment.

In all model calculations, we have assumed that variability in treatment effect is exclusively between-treatment variability. Two extensions that have been tested explicitly are effectvariability between the worms in a patient and between-patient variation of treatment effect (some patients systematically respond well, others poorly). Neither extension affects our conclusion or produced a better fit. The absence of between-patient variability justifies the use of the $\chi^{2}$ statistic for calculating the goodness-of-fit $(P)$ and determining CIs, the $\chi^{2}$ assuming independence between successive observations on the same persons.

Our conclusions have important implications for the public health impact of strategies based on annual ivermectin treatment, which is the currently recommended regimen. In earlier studies, we emphasized the potential of the drug for reducing the burden of blindness $[20,32]$. However, lack of sufficient 
follow-up data at that time meant that an irreversible effect of treatment on adult worms could not be demonstrated. This was the main cause of doubt about the potential of ivermectin for transmission control and thus for our cautiousness in designating it as the successor of vector control.

Now this point of view merits reconsideration. If each treatment leads to an irreversible reduction of fecundity by $\sim 30 \%$, after five treatments, the loss will be $>80 \%$ (not counting reinfections). These results should stimulate the assessment of the impact of higher doses on the viability of adult parasites. Extrapolating to a longer period of annual treatment is less straightforward; not everyone will be treated (a coverage of $65 \%-70 \%$ would be excellent in routine health care), and transmission will continue (albeit on a lower level), causing new infections. Preliminary computer simulation studies indicate that, although the impact of long-term ivermectin regimens is more pronounced than previously expected, the parasite is unlikely to be eradicated within a period of 15 years of annual treatment in an area where it is endemic.

\section{Acknowledgments}

We thank E. M. Samba, director of OCP, and the staff of OCP and the National Oncho Team in Ghana for their support; T. Stijnen for statistical assistance; and C. D. Johnson for invaluable comments on the manuscript.

\section{References}

1. Awadzi K, Dadzie KY, Schulz-Key H, Haddock DRW, Gilles HM, Aziz MA. The chemotherapy of onchocerciasis X. An assessment of four single dose treatment regimes of MK-933 (ivermectin) in human onchocerciasis. Ann Trop Med Parasitol 1985; 79:63-78.

2. Greene BM, Taylor HR, Cupp EW, et al. Comparison of ivermectin and diethylcarbamazine in the treatment of onchocerciasis. $N$ Engl J Med $1985 ; 313: 133-8$.

3. Larivière $M$, Vingtain $P$, Aziz MA, et al. Double-blind study of ivermectin and diethylcarbamazine in African onchocerciasis patients with ocular involvement. Lancet $1985 ; 2: 174-7$.

4. Schul7-Key H, Klager S, Awadzi K, et al. Treatment of human onchocerciasis: the efficacy of ivermectin on the parasite. Trop Med Parasitol 1985;36(suppl II):20.

5. Duke BOL, Zea-Flores G, Muñoz B. The embryogenesis of Onchocerca volvulus over the first year after a single dose of ivermectin. Trop Med Parasitol 1991;42:175-80.

6. Duke BOL, Zea-Flores G, Castro J, Cupp EW, Muñoz B. Comparison of the effects of a single dose and four six-monthly doses of ivermectin on adult Onchocerca volvulus. Am J Trop Med Hyg 1991;45:1327.

7. Duke BOL, Zea-Flores G, Castro I, Cupp EW, Muñoz B. Effects of multiple monthly doses of ivermectin on adult Onchocerca volvulus. Am J Trop Med Hyg 1990;43:657-64.

8. Duke BOL, Pacqué MC, Muñoz B, Greene BM, Taylor HR. Viability of adult Onchocerca volvulus after six 2-weekly doses of ivermectin. Bull World Health Organ 1991;69:163-8.

9. Duke BOL, Zea Flores G, Castro J, Cupp EW, Muñoz B. Effects of threemonth doses of ivermectin on adult Onchocerca volvulus. Am J Trop Med Hyg 1992;46:189-94.
10. Chavasse DC, Post RJ, Lemoh PA, Whitworth JAG. The effect of repeated doses of ivermectin on adult female Onchocerca volvulus in Sierra Leone. Trop Med Parasitol 1992;43:256-62.

11. Chavasse DC, Post RJ, Davies JB, Whitworth JAG. Absence of sperm from the seminal receptacle of female Onchocerca volvulus following multiple doses of ivermectin. Trop Med Parasitol 1993;44:155-8.

12. Kläger $S$, Whitworth JAG, Post RJ, Chavasse DC, Downham MD. How long do the effects of ivermectin on adult Onchocerca volvulus persist. Trop Med Parsitol 1993;44:305-10.

13. World Health Organization Expert Committee on Onchocerciasis Control Onchoccrciasis and its control. WHO Tech Rep Ser 1995;852.

14. Remme J, Baker RHA, De Sole G, et al. A community trial of ivermectin in the onchocerciasis focus of Asubende, Ghana. 1. Effect on the microfilarial reservoir and the transmission of Onchocerca volvulus. Trop Med Parasitol 1989;40:367 74 .

15. De Sole G, Awadzi K, Remme J, et al. A community trial of ivermectin in the onchocerciasis focus of Asubende, Ghana. II. Adverse reactions. Trop Med Parasitol 1989;40:375-82.

16. Dadzie KY, Remme J, De Sole G. Changes in ocular onchocerciasis after two rounds of community-based ivermectin treatment in a holoendemic onchocerciasis focus. Trans $R$ Soc Trop Med Hyg 1991;85:267 71.

17. Alley ES, Plaisier AP, Boatin BA, et al. The impact of five years of annual ivermectin treatment on skin microfilarial loads in the onchocerciasis focus of Asubende, Ghana. Trans R Soc Trop Med Hyg 1994;88:581 4.

18. Remme J, Ba O, Dadzie KY, Karam M. A force-of-infection model for onchocerciasis and its application in the epidemiological evaluation of the Onchocerciasis Control Programme in the Volta River basin area. Bull WHO 1986; 64:667-81.

19. Plaisier AP, Van Oortmarssen GJ, Habbema JDF, Remme J, Alley ES. ONCHOSIM: a model and computer simulation program for the transmission and control of onchocerciasis. Comput Methods Programs Biomed 1990; 31:43-56.

20. Habbema JDF, Alley ES, Plaisier AP, Van Oortmarssen GJ, Remme JHF. Epidemiological modelling for onchocerciasis control. Parasitol Today $1992 ; 8: 99-103$.

21. Habbema JDF, Van Oormmarssen GI, Plaisier AP. The ONCHOSIM model and its use in decision support for river blindness control. In: Isham $V$, Medley GF, eds. Epidemic models: their structure and relation to data. Cambridge, UK: Cambridge University Press, in press.

22. Plaisier AP, Van Oortmarssen GJ, Remme J, Habbema JDF. The reproductive lifespan of Onchocerca volvulus in West African savanna. Acta Trop 1991; $48: 271-84$.

23. De Sole G, Remme J, Awadzi K, et al. Adverse reactions after large-scale treatment of onchocerciasis with ivermectin: combined results from eight community trials. Bull World Health Organ 1989;67:707-19.

24. Nelder JA, Mead R. A simplex method of function minimization. Computer J 1965; 7:308-12.

25. Albiez EJ, Walter G, Kaiser A, et al. Histological examination of onchocercomata after therapy with ivermcetin. Trop Med Parasitol 1988;39:939.

26. Awadzi K, Dadzic KY, Schulz-Key H, Gilles HM, Fulford AJ, Aziz MA. The chemotherapy of onchocerciasis XI. A double-blind comparative study of ivermectin, diethylcarbamazine, and placebo in human onchocerciasis in Northern Ghana. Ann Trop Med Parasitol 1986;80:43342.

27. Taylor HR, Murphy RP, Newland HS, et al. Comparison of the treatment of ocular onchocerciasis with ivermectin and diethylcarbamazine. Arch Ophthalmol 1986; 104:863-70.

28. Diallo S, Aziz MA, Larivière $M$, et al. A double-blind comparison of the cfficacy and safety of ivermectin and diethylcarbamazine in a placebo controlled study of Senegalese patients with onchocerciasis. Trans $\mathrm{R}$ Soc Trop Med Hyg 1986; $80: 927-34$ 
29. Greene BM, White AT, Newland HS, et al. Single dose therapy with ivermectin for onchocerciasis. Trans Assoc Am Phys 1987;C:131-8.

30. Schulz-Key H, Soboslay PT, Hoffmann WH. Ivermectin-facilitated immunity. Parasitol Today 1992;8:152-3.

31. Schulz-Key H, Karam M. Periodic reproduction of Onchocerca volvulus. Parasitol Today 1986;2:284-6.

32. Remme J, De Sole G, Dadzie KY, et al. Large scale ivermectin distribution and its epidemiological consequences. Acta Leiden 1990;59:177 -91.

\section{Appendix}

Here we describe the model that combines hypotheses $\mathrm{H}_{2}$ and $\mathrm{H}_{2}{ }^{\prime}$. By making the appropriate simplifications, the models for $\mathrm{H}_{1}$, $\mathrm{H}_{2}$, and $\mathrm{H}_{2}^{\prime}$ are obtained.

If $m$ denotes the mean fraction total fecundity loss and $v_{i j}$ is the effectiveness of treatment round $i(i=1, \ldots, 5)$ in person $j$, then fraction $v_{i j} m$ of the worms in person $j$ will permanently cease Mf production immediately after treatment $i$. $v_{i j}$ is a random variable, which for each treatment $i$ and each person $j$ is generated from a gamma probability distribution with a mean $=1.0$ and a variation coefficient equivalent to the effect variability. For female worms in person $j$ that do not lose fecundity after treatment $i$ (a fraction, $1-v_{i j} m$ ), the rate of Mf production $r_{i j k}$ of each worm $k$ at time $t$ after treatment is described by the following:

$$
\begin{aligned}
r_{i j k}(t) & =r_{i j k}^{0}(t) \times\left(1-v_{i j} d\right) \times\left(\frac{t}{v_{i j} T r}\right)^{\mathrm{s}} & & \text { for } t<v_{i j} T r . \\
& =r_{i j k}^{0}(t) \times\left(1-v_{i j} d\right) & & \text { for } t \geq v_{i j} T r .
\end{aligned}
$$

In this expression, $r_{i j k}^{0}$ is the rate of Mf production of worm $k$ without treatment. This basic Mf production depends on the age and the mating history of the worm. Parameter $d$ is the mean irreversible productivity reduction, $T r$ is the mean duration of the recovery period, and $s$ is the shape of the recovery. If $s>1$, then the increase in Mf production is initially slow and accelerates by the end of the recovery period. If $s=1$, then this increase is linear.

In case the random variable $v_{i j}$ would take such high values that $v_{i j} m$ or $v_{i j} d$ (or both) is $>1$, the products are truncated to 1 . However, given the estimates of the effect variability $(\sim 0.5$; table 1$)$, these situations are highly unlikely.

In case of repeated treatment $(i>1)$, the model does not allow that an ineffective treatment (which implies a short recovery period) accelerates the recovery of a previous (effective) treatment. In case of total treatment failure ( $3 \%$ of treatments), $v_{i j}=0$ and no $\mathrm{Mf}$ are killed. It is assumed that $\mathrm{Mf}$ will in principle be detectable in the skin immediately after their release from the worm. No provision is made for a delay due to dispersal in the body and penetration of skin tissues. Mf are assumed to have a fixed lifespan (Tm). In this simple concept, if the Mf production rate of the worms in a person stabilizes at time $t$, then the Mf density in the skin stabilizes at time $t+T m$. 